\title{
Novel concepts in airway inflammation and remodelling in asthma
}

\author{
Sejal Saglani ${ }^{1,2}$ and Clare M. Lloyd ${ }^{1}$
}

\author{
Affiliations: \\ ${ }^{1}$ Inflammation, Repair and Development Section, National Heart \& Lung Institute, Imperial College London, \\ London, UK. \\ ${ }^{2}$ Dept of Respiratory Paediatrics, Royal Brompton Hospital, London, UK.
}

\section{Correspondence:}

Sejal Saglani, 368 Sir Alexander Fleming Building, Imperial College London, Exhibition Road, London SW7 2AZ, UK.

E-mail: s.saglani@imperial.ac.uk

ABSTRACT The hallmark pathological features of asthma include airway eosinophilic inflammation and structural changes (remodelling) which are associated with an irreversible loss in lung function that tracks from childhood to adulthood. In parallel with changes in function, pathological abnormalities occur early, during the pre-school years, are established by school age and subsequently remain (even though symptoms may remit for periods during adulthood). Given the equal importance of inflammation and remodelling in asthma pathogenesis, there is a significant disparity in studies undertaken to investigate the contribution of each. The majority focus on the role of inflammation, and although novel therapeutics such as those targeted against T-helper cell type 2 (Th2) mediators have arisen, it is apparent that targeting inflammation alone has not allowed disease modification. Therefore, unless airway remodelling is addressed for future therapeutic strategies, it is unlikely that we will progress towards a cure for asthma. Having acknowledged these limitations, the focus of this review is to highlight the gaps in our current knowledge about the mechanisms underlying airway remodelling, the relationships between remodelling, inflammation and function, remodelling and clinical phenotypes, and the importance of utilising innovative and realistic pre-clinical models to uncover effective, disease-modifying therapeutic strategies.

$@$ ERSpublications

We discuss mechanisms of airway inflammation and remodelling in asthma to uncover effective therapeutic strategies http://ow.ly/SsXiO 


\section{Novel ideas and hypotheses}

1) Not all patients have similar changes in each parameter of airway remodelling. Remodelling phenotypes should therefore be delineated, i.e. the contribution of each structural change (reticular basement membrane thickness, airway smooth muscle mass, angiogenesis) in an individual patient needs to be assessed. Subsequently, together with the clinical phenotype and inflammatory phenotype, the most appropriate personalised therapy can be identified for the individual.

2) Unbiased statistical approaches that serve to identify phenotypes and endotypes need to include structural airway changes, in a similar manner to recent analyses that have included eosinophilic and neutrophilic inflammation.

3) Clinical trials should incorporate airway biopsies and assessments of airway remodelling as outcome measures.

4) The remodelling phenotype of noneosinophilic, noninflamed and T-helper type 2 (Th2)-low patients needs to be investigated to allow discovery of effective molecular therapies.

\section{Introduction}

The hallmark pathological features of asthma include airway eosinophilic inflammation and structural changes (remodelling) characterised by increased thickness of the subepithelial reticular basement membrane (RBM), increased airway smooth muscle (ASM) mass, angiogenesis and goblet cell hyperplasia which are associated with an irreversible loss in lung function that tracks from childhood to adulthood [1]. The structural airway changes are apparent in asthma even in mild disease [2]; however, the degree of remodelling often worsens with increasing disease severity [3]. In addition, the onset of the pathological changes occurs early, during the pre-school years $[4,5]$, and they are established by school age and subsequently remain (even though symptoms may remit for periods during adulthood) [6].

Given the importance of both inflammation and remodelling in asthma pathogenesis, there is a significant disparity in the number of studies that have investigated the contribution of each. Most studies, both clinical and mechanistic, focus on inflammatory parameters alone. Several factors contribute to this bias and assessments of remodelling cannot be made noninvasively at present since the gold standard remains tissue analysis which requires bronchial biopsy. As a result, longitudinal assessments especially following interventions in clinical trials are not practical and therefore are rarely included as outcome measures. Although imaging with computed tomography (CT) scans can be used, the data is variable and currently CT scans are not a reliable surrogate for tissue assessments. However, biopsies are usually only representative of proximal airways as most are endobronchial and taken under direct vision; structural changes in the peripheral airways therefore remain only minimally investigated. Even when pre-clinical studies are considered, few models investigate mechanisms of airway remodelling, perhaps because remodelling assessments require chronic and long-term models.

Having acknowledged these limitations, the main focus of this review will be to highlight the gaps in our current knowledge relating to airway remodelling in asthma, and to identify future approaches that will allow better understanding of the relationships between remodelling and function, potential relationships with clinical phenotypes, and the importance of utilising innovative and realistic pre-clinical models to discover novel therapies [7] targeted to structural airway changes. Overall, therapies to date that have targeted inflammation alone have not resulted in disease modification. A useful strategy may therefore be to give airway remodelling equal importance for future therapeutics in order to progress towards disease-modifying therapies in asthma.

\section{The need to recognise remodelling phenotypes in addition to clinical and inflammatory phenotypes in asthma}

It is accepted that asthma is a heterogeneous disease, with numerous factors such as age of onset, disease severity, allergic status and environmental exposures contributing to clinical manifestation [8]. Although unbiased analyses to identify clinical phenotypes have been extensively undertaken, and many of these approaches have included inflammatory parameters, especially eosinophilic inflammation $[9,10]$, assessments of airway remodelling have yet to be incorporated. It is acknowledged that currently available molecular therapies, including novel monoclonal antibodies, target inflammatory mediators and are successful in patients with a T-helper cell type 2 (Th2)-predominant profile [11], but therapies for non-Th2-high or noninflammatory phenotypes are needed [12].

It is certain that future therapeutic strategies for asthma will incorporate phenotype-specific approaches as we head towards a personalised approach to treating asthma $[11,13]$, but to allow this to be successful, it is essential that we include both remodelling and inflammatory markers in analyses. We need to accept that asthma incorporates a clinical phenotype, inflammatory phenotype and remodelling phenotype, and the interactions and contributions from each facet will determine the most effective therapy in the 
individual patient (figure 1). An example of the use of structural airway changes to identify clusters or phenotypes was undertaken using high-resolution CT scans in adults [14]. This allowed distinction of patients into clusters according to their bronchial wall volume or bronchial lumen volume and revealed one specific cluster had very little evidence of bronchial wall changes that would be compatible with airway remodelling on CT. This is an interesting finding as it is usually assumed that all patients with asthma have airway remodelling. Although as a group, patients with asthma have evidence of airway remodelling $[4,15]$, it is important to note that in a similar manner to inflammation, the degree to which each structural change is present in an individual can be very variable, often with marked overlap between asthmatics and controls [15]. An interesting avenue for future investigation would be to define the degree of each structural airway change in a patient to see whether there is concordance; so does a very thickened basement membrane associate with a large change in ASM in the same patient? When phenotypes of severe asthma in adults are related to pathology, it has been shown that many with adult-onset disease have little evidence of eosinophilic inflammation and also have little evidence of airway remodelling [16]. This may be because of the effect of maintenance steroid therapy. However, this observation is important and requires additional confirmation because it has been proposed that most asthma can be divided into early (childhood) or late (adult) onset [17, 18]. Inflammation and remodelling of early-onset disease has been extensively studied in both children and adults. It is predominantly atopic and eosinophilic, and associated with marked airway remodelling, including increased RBM thickness and ASM mass [15, 19]. These features persist despite high-dose maintenance steroid therapy, suggesting the pathology is relatively steroid resistant. In contrast, although many clinical and inflammatory features of late-onset disease are reported [20], little is known about the underlying airway remodelling of this phenotype even though up
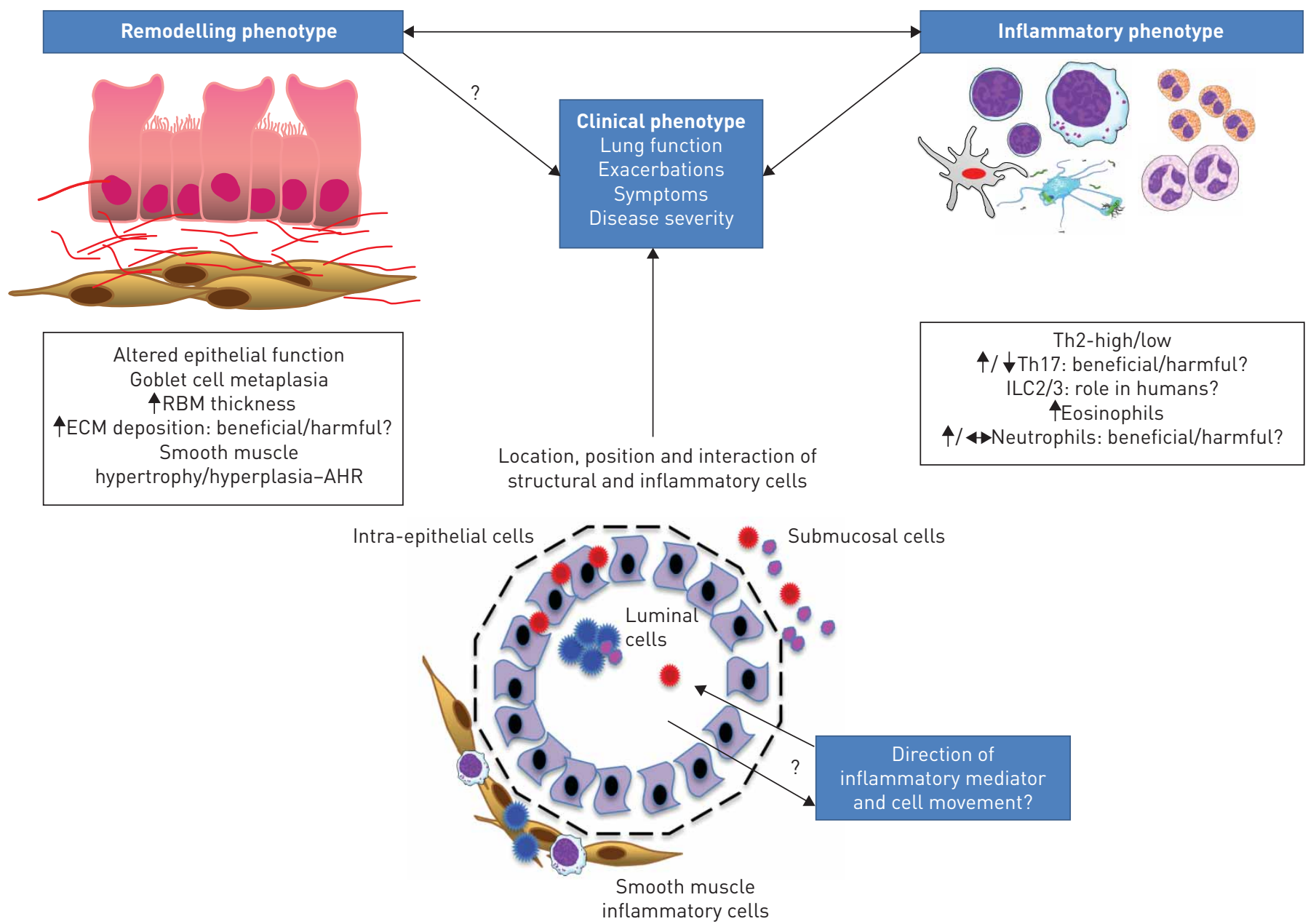

FIGURE 1 Interactions between remodelling, clinical and inflammatory phenotypes determine disease manifestation and the optimal molecular targets for intervention in the individual patient. Remodelling and inflammatory phenotypes may directly influence each other, and the location of inflammatory cells in relation to structural cells is a critical determinant of downstream effects, including mediator release and lung function. However, both remodelling and inflammation may also directly influence clinical manifestation of disease independently of each other. RBM: reticular basement membrane; ECM; extracellular matrix; AHR: airway hyperresponsiveness; Th: T-helper cell; ILC2/3: innate lymphoid cell group 2/3. 
to one-third of adults with asthma are affected. It could be hypothesised that the CT cluster that had little evidence of bronchial wall changes [14] may correspond to this late-onset phenotype.

\section{Limitations of therapy targeted at inflammation}

Currently, the "molecular" approach to asthma management is based predominantly on inflammatory parameters; however, this may not be the optimal approach as between 30 and $48 \%$ of patients change their physiological or inflammatory phenotype, regardless of disease severity, within 1 year [21]. Phenotype instability when defined according to inflammation is not specific to adults. Children with severe asthma also have significant inflammatory phenotype instability changing between eosinophilic, neutrophilic, lymphocytic and paucigranulocytic randomly without any predictors within a 1-year period [22]. Similarly, in adults on inhaled steroid therapy, the sputum inflammatory profile commonly changed in subjects with both moderate and severe asthma over 1 year, and only one-third of subjects had a stable phenotype [23]. Given the relative instability of airway inflammation in asthma and the added complication of changes in inflammation during exacerbations [24], it is unlikely that management based on inflammatory phenotype alone is sufficient. An interesting point about the subset of patients that do have persistently noneosinophilic disease is their poor response to inhaled steroids [25]. Even though many of these patients have mild-to-moderate asthma, there is a lack of effective maintenance therapies. However, both eosinophilic and noneosinophilic patients appear equally responsive to bronchodilators such as salbutamol [25], suggesting ASM remodelling is perhaps present in both. However, currently, nothing is known about the remodelling phenotype of these persistent noneosinophilic patients, thus preventing application of appropriately targeted therapies.

Another phenotypic distinction that has been described in asthma is that some patients have Th2-high, whilst others have Th2-low asthma [26]. Although the inflammatory profile of Th2-high asthma is eosinophilic and Th2-low disease is characterised by an absence of eosinophilia, it remains uncertain whether associated underlying structural airway changes are also distinct in these two categories. The implications are important as Th2-high disease is typically steroid responsive, but Th2-low disease is not and therefore can be harder to treat. Most recent novel therapeutics for severe asthma have successfully targeted Th2 mediators (anti-interleukin (IL)-5, anti-IL-13 antibody therapy [27, 28]) or Th2 cells [29]. It is possible that Th2-low patients will require therapies that are specifically targeted to structural changes rather than inflammation, but this has yet to be shown. However, in support of this hypothesis, adults with severe steroid-resistant asthma have increased circulating fibrocytes (progenitors of mesenchymal cells that are less responsive to steroids) compared with patients with milder disease [30].

\section{Relationships between airway inflammation and remodelling: do any exist?}

Until recently, it was thought that chronic inflammation may drive remodelling, but increasingly this proposition has been disputed. Studies in children have been the strongest to refute the notion that inflammation causes structural airway changes. Children aged between 6 and 16 years with severe asthma already have evidence of marked eosinophilic inflammation and remodelling [15]. Moreover, even younger pre-school children (median age 3 years) with severe recurrent wheezing have evidence of increased RBM thickness and tissue eosinophilic inflammation [5]. Interestingly, a recent biopsy study in pre-school children with severe wheeze has shown the presence of several features of remodelling, including increased RBM thickness, ASM, vascularity and mucus gland area, but no relationship between inflammatory cell counts in biopsies and remodelling [31]. A limitation of human studies, especially paediatric studies, is the relative difficulty in obtaining repeated invasive biopsies to assess longitudinal changes in pathology over time. However, evidence that the pathophysiological abnormalities of asthma, inflammation, remodelling and airway hyperresponsiveness (AHR) develop in parallel is apparent from a murine model of early-onset, neonatal (day 3 of life) inhaled allergen-induced airways disease, in which inflammation and remodelling occurred in parallel and were not sequential [32].

\section{Are eosinophilic inflammation and remodelling linked?}

Targeting eosinophilic inflammation in adult asthma is successful in reducing exacerbations [33]. However, experimental models using eosinophil knockout mice demonstrate similar allergic airways disease to wild-type mice [34]. Moreover, biopsy studies of infants with severe wheeze [35-37] have shown that eosinophils are not necessary for the development of asthma. In addition, targeting eosinophils does not seem to modify disease progression, as development of asthma from pre-school wheeze is not prevented by steroids $[38,39]$, nor is there a maintained disease-modifying effect after anti-IL- 5 antibody therapy is stopped [40]. Therefore, eliminating eosinophilic inflammation neither prevents the development of asthma nor does it modify the reduction in lung function that occurs. The relatively weak relationship between eosinophilic airway inflammation and lung function is apparent from clinical trials of the monoclonal antibody to IL-5 that reduces eosinophil recruitment. Use of anti-IL-5 antibody (mepolizumab) resulted in an improvement in asthma exacerbations, but had no effect on lung function 
[28]. Similarly, strategies that reduce eosinophilic inflammation by targeting sputum eosinophils result in reduced exacerbations, but have no impact on lung function [33]. Interestingly, anti-IL-5 antibody has been shown to reduce protein deposition within the subepithelial RBM in mild-to-moderate asthma, in conjunction with a reduction in tissue eosinophil numbers [41]. Indeed, a positive relationship between biopsy eosinophils and thickness of the RBM has also been reported in pre-school children with recurrent wheeze [5]. In addition, adult patients with prior occupational asthma, in clinical remission, still have evidence of persistent increased RBM thickness and eosinophilic inflammation [6,42]. Therefore, although there is evidence for a relationship between eosinophilic inflammation and RBM thickness, a relationship between eosinophilic inflammation and lung function or persistent symptoms is less convincing.

An interesting disconnect between eosinophilic inflammation and remodelling was demonstrated in an adult biopsy study that used different inhalation challenges and assessed impact on inflammation and remodelling. The effects of allergen (to induce inflammation and bronchoconstriction) were compared with methacholine (bronchoconstriction alone) or saline (control) in adults with asthma. As expected, allergen challenge induced all pathophysiological abnormalities, including bronchoconstriction, eosinophilic inflammation and remodelling. Strikingly, however, methacholine challenge induced only bronchoconstriction and remodelling without inflammation [43]. Data from experimental models also suggest a much tighter relationship between airway contraction, lung function and remodelling than any association between inflammation and remodelling [44]. In late-onset (adult) murine models it is becoming increasingly apparent that remodelling can develop despite minimal or absent airway inflammation [45]. Using a genetically susceptible mouse model, with bronchial epithelial overexpression of Smad2 and chronic inhalation of ovalbumin, without peripheral sensitisation, marked AHR and remodelling developed in the absence of airway inflammation. The remodelling was characterised by increased ASM and peribronchiolar collagen deposition, and was mediated by the epithelial cytokine endothelin-1 [45]. A reversal of the remodelling was achieved using an antibody to endothelin-1 and also resulted in an abrogation of AHR. In another adult model of inhaled house dust mite (HDM) exposure, eosinophil knockout ( $\triangle$ dblGATA) mice developed both AHR and remodelling to the same extent as wild-type mice [34]. These data show that remodelling can develop independently of inflammation, but seems more closely related to lung function. Indeed, the loss in lung function seen in asthma [1] is proposed to result from airway remodelling, not inflammation, but despite this, therapeutic targets for remodelling remain scarce.

The role of neutrophils in asthma and relationships with remodelling

It is hypothesised that, in the context of asthma, neutrophils are pathogenic and are associated with more severe disease [46]. However, this is becoming increasingly less certain. For example, sputum neutrophilic inflammation is not a marker of disease severity $[47,48]$. In fact, it may be that neutrophils play a protective or regulatory role. They are increased during infection induced exacerbations, yet use of the anti-inflammatory macrolide azithromycin has proved disappointing $[49,50]$. Neutrophilic inflammation is particularly associated with Th17 responses and disease severity in asthma [51]. However, much of these data come from murine models that have used ovalbumin and therefore translation to patients is questionable. Exposure of HDM and diesel exhaust particles (DEPs) in a murine model resulted in pulmonary neutrophilia with DEP exposure alone, but co-exposure of both HDM and DEPs caused a predominant eosinophilia [52]. Importantly, clinical trials of an anti-IL-17 receptor antibody in patients with poorly controlled asthma have shown no benefit [53]. Data from children with severe asthma suggests levels of IL-17A are elevated in bronchoalveolar lavage and induced by steroids [54], but do not support a pathogenic role.

A relationship between neutrophilic inflammation and remodelling is less apparent than between eosinophilic inflammation and remodelling. However, a relationship between matrix metalloproteinase (MMP)-9 (a mediator known to be involved in airway remodelling) and neutrophils is being increasingly recognised. Allergen stimulation of peripheral leukocytes from adults with asthma resulted in release of MMP-9 only from neutrophils, not eosinophils [55]. Relationships between established remodelling or lung function were not assessed. However, in a separate study of severe asthmatics in which CT scans were used to assess airway lumen and wall narrowing, groups were stratified according to airway neutrophils. Highest levels of MMP-9 were found in patients with highest neutrophils, independently of airway geometry. Interestingly, however, high MMP-9 and high neutrophils also related to an increased airway luminal area, suggesting the patients with these features had significant airway dilatation and bronchiectasis [56]. A previous report in severe asthma from the same group had also shown a relationship specifically between airway neutrophils and geometry on CT scan, not with airway eosinophils [14]. Another study in smokers with severe asthma has shown a relationship between low sputum MMP-9/TIMP (tissue inhibitors of metalloproteinase) ratio, persistent airflow obstruction and reduced airway lumen on CT [57]. However, a post mortem study of fatal asthma patients showed increased smooth muscle was only associated with airway eosinophilia, but not neutrophilia [58]. It is therefore apparent that more assessments of airway structure and mechanistic studies 
are needed to better understand the relationship between neutrophils, MMP-9, lung function and remodelling, especially if MMP-9 might be a potential therapeutic target for neutrophil predominant disease.

\section{Targeting remodelling to achieve disease modification: potential cells and mediators of interest \\ Bronchial epithelium}

The bronchial epithelium is increasingly recognised as a critical airway cell involved in the pathogenesis and persistence of asthma [59]. This is highlighted by the altered function of the epithelium in patients with asthma compared with those without [60]. Epithelial cells actively secrete immune mediators in response to environmental stimuli [61], which are different in patients with asthma compared with controls [62]. Asthmatic epithelium also has altered barrier function [63], and is a critical bridge between external environmental exposures and pulmonary inflammatory and immune responses [64]. Bronchial epithelial cells dictate downstream responses, despite being structural components of the airway wall and not inflammatory or immune cells. Their intimate link with the development of airway remodelling has been highlighted by the hypothesis of epithelial-to-mesenchymal transition causing airway remodelling in parallel with inflammatory responses [65].

In addition, bronchial epithelial cell function is altered in pre-school wheezers [66], and although it is uncertain whether this predicts progression to asthma, given the evidence of abnormal epithelial function in children and adults with established asthma [67], and the identification of genes associated with asthma being expressed in the bronchial epithelium [68,69], this is definitely an important cell that should be further investigated as a target for disease modification. A further advantage is its direct accessibility via the inhaled route.

\section{Airway smooth muscle}

The role of other structural airway cells as key regulators of asthma has been less thoroughly investigated. ASM cells are increased in both number and size $[19,70]$ in asthma, but they are not solely involved in airway contraction. They demonstrate active secretion of inflammatory mediators and are intimately associated with the extracellular matrix, and both their secretory and proliferative capacity is directly affected by the surrounding matrix [71]. Not only is the relationship between ASM and matrix important in determining function, but equally it has been proposed that the presence of inflammatory cells, specifically mast cells, within ASM bundles is pathognomic of asthma [72]. Cross-talk between mast cells and ASM has been shown to directly induce AHR via IL-33 [73]. Bitter taste receptors have been highlighted as a potential novel target for asthma as they were found to be expressed on murine ASM and their agonists caused smooth muscle relaxation [74]. Transcriptomic profiling has shown they are upregulated in peripheral blood from severe asthmatic children [75]. Subsequently, it has become apparent that bitter taste receptors are expressed on several other pulmonary cell types, including epithelial cells, lymphocytes and macrophages, and are therefore involved in various functions, including ciliary beating, muscle relaxation and production of inflammatory mediators [76]. Encouragingly, a recent interventional clinical trial was designed with the aim of assessing the impact of a calcium channel blocker on established airway remodelling. Specifically, the primary outcome measure was change in bronchial smooth muscle area after 12 months of treatment with the calcium channel blocker gallopamil in adults with severe asthma [77]. Treatment resulted in a reduction in bronchial smooth muscle area and in exacerbations after the intervention period. This trial highlights the importance of addressing remodelling, but also demonstrates that a longer duration of intervention and serial biopsies are needed to see an effect on structural airway cells. However, it is extremely promising to see that therapeutics targeting remodelling can reverse established changes in severe disease and we do not need to rely on focussing therapeutics for remodelling in very early disease before the changes are established.

Of all the pathological parameters that might affect the natural history of asthma, current data suggests the function of structural airway cells, including ASM and epithelium, may be the most important. Pre-school children with severe wheezing that developed asthma only had significantly more ASM in their endobronchial biopsies at pre-school age compared with those wheezers that did not develop asthma, while tissue eosinophilia or RBM thickness were not predictors [36]. In addition, increased ASM was the only change associated with atopy in severe pre-school wheezers [31]. Thus, early abnormalities in ASM function may determine asthma development. However, nothing is known about ASM function in infants or pre-school children.

\section{The need for complex, clinically relevant models to identify therapeutic targets for airway remodelling}

In order to identify novel therapeutics to target remodelling, several factors need to be considered. First, pre-clinical models that are translatable and truly reflect patient phenotypes must be used [7]. This means complex models need to be developed. An example is the recent development of a mouse model in which features of allergic airways disease persisted for up to 6 months after allergen exposure had ceased [78]. Not only can this be used to study factors underlying disease remission, but also to understand disease 
persistence. Human data has shown that even in the absence of symptoms pathological changes, specifically structural changes, persist in the airways [79] and the mechanisms contributing to this can now be examined. The complexity of factors causing disease persistence was demonstrated in this chronic model as three comparator allergen exposures (ragweed, Alternaria and HDM) and knockout of two separate lymphoid cell types (T-cells and innate lymphoid cells) were used. Multiple feedback and feed-forward circuits between innate lymphoid cells, epithelial cells, IL-33 and IL-13 were identified, but the key clinical message was the importance of IL-33 in maintaining disease, not in inception [78]. A similar message arose from translational work using a neonatal mouse model and airway samples from children with severe therapy-resistant asthma. Although IL-13 was difficult to detect in these patients on high-dose steroid therapy [15], tissue expression of IL-33 was elevated. Assessment of the effects of therapeutic inhaled budesonide in vivo in the murine model revealed a reduction in IL-13 levels, but IL-33 was unaffected. These data complemented the biopsy findings and revealed IL-33 as a steroid-resistant mediator of specific interest in severe disease. Importantly, the mouse data also showed intranasal administration of IL-33 resulted in increased lung collagen levels, and there was a positive correlation between subepithelial RBM thickness and IL-33 expression in biopsies from children. Finally, stimulation of primary fibroblasts from asthmatic patient biopsies with IL-33 resulted in collagen secretion which was maintained despite steroids [80]. These data also highlighted IL-33 as a novel target for established severe asthma (disease persistence), and showed blocking its action may overcome steroid resistance in severe disease and impact airway remodelling. Both of the studies have highlighted a therapeutic role for IL-33, but have used complex murine models combined with data from patients. There is evidence from experimental models that molecules such as IL-25, activin A and transforming growth factor (TGF)- $\beta$ are involved in the induction of airway remodelling $[44,45]$, and that blocking their action improves both airway remodelling and lung function. However, these are also complex models that involve altered tissue-specific (epithelial) gene expression, not simple knockout mice. Activation of the molecules within the TGF- $\beta$ superfamily was also identified in patients with mild asthma following allergen challenge, whereby activin receptor-positive cells increased in both the epithelium and submucosa after allergen challenge, and bone morphogenic proteins and activin-like kinase were downregulated [81], suggesting modulation in this signalling pathway may contribute to remodelling. However, this was only detected by using a complex human model of in vivo allergen challenge.

\section{Application of unbiased techniques to uncover mechanisms of remodelling}

The application of transcriptomic profiling to bronchial epithelial cells allowed the discovery of the Th2-high and -low gene signature in adult patients, which translated to a clinical response to anti-IL-13 antibody therapy [27]. In a similar manner, proteomic profiling of endobronchial biopsies from asthmatics was used to determine predictors of response to anti-IgE antibody omalizumab. This revealed galectin-3, which is also an extracellular matrix protein involved in remodelling, as a biomarker of response [82]. This protein can be measured in urine and serum, and could be tested in future studies as a marker of response to therapy [83]. A proposed future strategy to allow the complexities and heterogeneity underlying the pathophysiology of asthma, with numerous dysregulated molecular pathways to be disentangled, is to utilise unbiased systems biology.

\section{Summary}

Inflammation and remodelling are critical components of the pathophysiology of asthma. Although both contribute significantly to disease pathogenesis, to date mechanistic studies and drug discovery have focussed on inflammatory targets. Although these novel therapeutics (predominantly monoclonal antibodies to $\operatorname{IgE}$ and Th2 cytokines IL-5 and IL-13) have achieved improvements in disease control, they are only applicable to a subgroup of patients and do not result in disease modification. We suggest that to make a step-change in asthma therapy, the focus of research now needs to be on investigating mechanisms underlying Th2-low phenotypes and airway remodelling, and the approaches used will have to reflect disease heterogeneity, and include complex experimental approaches with in vitro, in vivo, animal and human studies.

\section{References}

1 Tai A, Tran H, Roberts M, et al. The association between childhood asthma and adult chronic obstructive pulmonary disease. Thorax 2014; 69: 805-810.

2 Wilson SJ, Rigden HM, Ward JA, et al. The relationship between eosinophilia and airway remodelling in mild asthma. Clin Exp Allergy 2013; 43: 1342-1350.

3 Bonsignore MR, Profita M, Gagliardo R, et al. Advances in asthma pathophysiology: stepping forward from the Maurizio Vignola experience. Eur Respir Rev 2015; 24: 30-39.

4 Barbato A, Turato G, Baraldo S, et al. Epithelial damage and angiogenesis in the airways of children with asthma. Am J Respir Crit Care Med 2006; 174: 975-981.

5 Saglani S, Payne DN, Zhu J, et al. Early detection of airway wall remodeling and eosinophilic inflammation in preschool wheezers. Am J Respir Crit Care Med 2007; 176: 858-864.

6 Sumi Y, Foley S, Daigle S, et al. Structural changes and airway remodelling in occupational asthma at a mean interval of 14 years after cessation of exposure. Clin Exp Allergy 2007; 37: 1781-1787. 
7 Holmes AM, Solari R, Holgate ST. Animal models of asthma: value, limitations and opportunities for alternative approaches. Drug Discov Today 2011; 16: 659-670.

8 Divekar R, Calhoun WJ. Heterogeneity of asthma in society. Adv Exp Med Biol 2014; 795: 31-41.

9 Ray A, Oriss TB, Wenzel SE. Emerging molecular phenotypes of asthma. Am J Physiol Lung Cell Mol Physiol 2015; 308: L130-L140.

10 Wenzel SE. Asthma phenotypes: the evolution from clinical to molecular approaches. Nat Med 2012; 18: 716-725.

11 Fajt ML, Wenzel SE. Asthma phenotypes and the use of biologic medications in asthma and allergic disease: the next steps toward personalized care. J Allergy Clin Immunol 2015; 135: 299-310.

12 Gelfand EW, Alam R. The other side of asthma: steroid-refractory disease in the absence of $\mathrm{T}_{\mathrm{H}} 2$-mediated inflammation. J Allergy Clin Immunol 2015; 135: 1196-1198.

13 Gonem S, Desai D, Siddiqui S, et al. Evidence for phenotype-driven treatment in asthmatic patients. Curr Opin Allergy Clin Immunol 2011; 11: 381-385.

14 Gupta S, Hartley R, Khan UT, et al. Quantitative computed tomography-derived clusters: redefining airway remodeling in asthmatic patients. J Allergy Clin Immunol 2014; 133: 729-738.

15 Bossley CJ, Fleming L, Gupta A, et al. Pediatric severe asthma is characterized by eosinophilia and remodeling without $\mathrm{T}_{\mathrm{H}} 2$ cytokines. J Allergy Clin Immunol 2012; 129: 974-982.

16 Miranda C, Busacker A, Balzar S, et al. Distinguishing severe asthma phenotypes: role of age at onset and eosinophilic inflammation. J Allergy Clin Immunol 2004; 113: 101-108.

17 Amelink M, de Groot JC, de Nijs SB, et al. Severe adult-onset asthma: a distinct phenotype. J Allergy Clin Immunol 2013; 132: 336-341.

18 Amelink M, de Nijs SB, de Groot JC, et al. Three phenotypes of adult-onset asthma. Allergy 2013; 68: 674-680.

19 Regamey N, Ochs M, Hilliard TN, et al. Increased airway smooth muscle mass in children with asthma, cystic fibrosis, and non-cystic fibrosis bronchiectasis. Am J Respir Crit Care Med 2008; 177: 837-843.

20 Tan DJ, Walters EH, Perret JL, et al. Age-of-asthma onset as a determinant of different asthma phenotypes in adults: a systematic review and meta-analysis of the literature. Expert Rev Respir Med 2015; 9: 109-123.

21 Kupczyk M, Dahlén B, Sterk PJ, et al. Stability of phenotypes defined by physiological variables and biomarkers in adults with asthma. Allergy 2014; 69: 1198-1204.

22 Fleming L, Tsartsali L, Wilson N, et al. Sputum inflammatory phenotypes are not stable in children with asthma. Thorax 2012; 67: 675-681.

23 Al-Samri MT, Benedetti A, Préfontaine D, et al. Variability of sputum inflammatory cells in asthmatic patients receiving corticosteroid therapy: a prospective study using multiple samples. J Allergy Clin Immunol 2010; 125: 1161-1163.

24 Wang F, He XY, Baines KJ, et al. Different inflammatory phenotypes in adults and children with acute asthma. Eur Respir J 2011; 38: 567-574.

25 McGrath KW, Icitovic N, Boushey HA, et al. A large subgroup of mild-to-moderate asthma is persistently noneosinophilic. Am J Respir Crit Care Med 2012; 185: 612-619.

26 Fahy JV. Type 2 inflammation in asthma - present in most, absent in many. Nat Rev Immunol 2015; 15: 57-65.

27 Corren J, Lemanske RF, Hanania NA, et al. Lebrikizumab treatment in adults with asthma. N Engl J Med 2011; 365: 1088-1098.

28 Pavord ID, Korn S, Howarth P, et al. Mepolizumab for severe eosinophilic asthma (DREAM): a multicentre, double-blind, placebo-controlled trial. Lancet 2012; 380: 651-659.

29 Krug N, Hohlfeld JM, Kirsten AM, et al. Allergen-induced asthmatic responses modified by a GATA3-specific DNAzyme. N Engl J Med 2015; 372: 1987-1995.

30 Lo CY, Michaeloudes C, Bhavsar PK, et al. Increased phenotypic differentiation and reduced corticosteroid sensitivity of fibrocytes in severe asthma. J Allergy Clin Immunol 2015; 135: 1186-1195.

31 Lezmi G, Gosset P, Deschildre A, et al. Airway remodeling in preschool children with severe recurrent wheeze. Am J Respir Crit Care Med 2015; 192: 164-171.

32 Saglani S, Mathie SA, Gregory LG, et al. Pathophysiological features of asthma develop in parallel in house dust mite-exposed neonatal mice. Am J Respir Cell Mol Biol 2009; 41: 281-289.

33 Green RH, Brightling CE, McKenna S, et al. Asthma exacerbations and sputum eosinophil counts: a randomised controlled trial. Lancet 2002; 360: 1715-1721.

34 Fattouh R, Al-Garawi A, Fattouh $\mathrm{M}$, et al. Eosinophils are dispensable for allergic remodeling and immunity in a model of house dust mite-induced airway disease. Am J Respir Crit Care Med 2011; 183: 179-188.

35 Malmstrom K, Pelkonen AS, Makela MJ. Remodeling, inflammation and airway responsiveness in early childhood asthma. Curr Opin Allergy Clin Immunol 2013; 13: 203-210.

36 O’Reilly R, Ullmann N, Irving S, et al. Increased airway smooth muscle in preschool wheezers who have asthma at school age. J Allergy Clin Immunol 2013; 131: 1024-1032.

37 Saglani S, et al. Airway remodeling and inflammation in symptomatic infants with reversible airflow obstruction. Am J Respir Crit Care Med 2005; 171: 722-727.

38 Bisgaard $\mathrm{H}$, Hermansen MN, Loland L, et al. Intermittent inhaled corticosteroids in infants with episodic wheezing. N Engl J Med 2006; 354: 1998-2005.

39 Guilbert TW, Morgan WJ, Zeiger RS, et al. Long-term inhaled corticosteroids in preschool children at high risk for asthma. N Engl J Med 2006; 354: 1985-1997.

40 Haldar P, Brightling CE, Singapuri A, et al. Outcomes after cessation of mepolizumab therapy in severe eosinophilic asthma: a 12-month follow-up analysis. J Allergy Clin Immunol 2014; 133: 921-923.

41 Flood-Page P, Menzies-Gow A, Phipps S, et al. Anti-IL-5 treatment reduces deposition of ECM proteins in the bronchial subepithelial basement membrane of mild atopic asthmatics. J Clin Invest 2003; 112: 1029-1036.

42 Maestrelli P, Saetta M. Recovery from adult-onset asthma and airway remodelling. Clin Exp Allergy 2007; 37: $1733-1735$.

43 Grainge CL, Lau LC, Ward JA, et al. Effect of bronchoconstriction on airway remodeling in asthma. N Engl J Med 2011; 364: 2006-2015.

44 Gregory LG, Mathie SA, Walker SA, et al. Overexpression of Smad2 drives house dust mite-mediated airway remodeling and airway hyperresponsiveness via activin and IL-25. Am J Respir Crit Care Med 2010; 182: 143-154.

45 Gregory LG, Jones CP, Mathie SA, et al. Endothelin-1 directs airway remodeling and hyper-reactivity in a murine asthma model. Allergy 2013; 68: 1579-1588. 
Ciepiela O, Ostafin M, Demkow U. Neutrophils in asthma - a review. Respir Physiol Neurobiol 2015; 209: 13-16. Rossall M, Cadden P, Kolsum U, et al. A comparison of the clinical and induced sputum characteristics of earlyand late-onset asthma. Lung 2012; 190: 459-462.

Hinks TS, Zhou X, Staples KJ, et al. Innate and adaptive T cells in asthmatic patients: relationship to severity and disease mechanisms. J Allergy Clin Immunol 2015; 136: 323-333.

Brusselle GG, Vanderstichele C, Jordens P, et al. Azithromycin for prevention of exacerbations in severe asthma (AZISAST): a multicentre randomised double-blind placebo-controlled trial. Thorax 2013; 68: 322-329.

ong EH, Porter JD, Edwards MR, et al. The role of macrolides in asthma: current evidence and future directions. Lancet Respir Med 2014; 2: 657-670.

F, Mahay G, et al. IL-17 in severe asthma. Where do we stand? Am J Respir Crit Care Med 2014; 190: 1094-1101.

Brandt EB, Kovacic MB, Lee GB, et al. Diesel exhaust particle induction of IL-17A contributes to severe asthma. J Allergy Clin Immunol 2013; 132: 1194-1204.

Busse WW, Holgate S, Kerwin E, et al. Randomized, double-blind, placebo-controlled study of brodalumab, a human anti-IL-17 receptor monoclonal antibody, in moderate to severe asthma. Am J Respir Crit Care Med 2013; 188: 1294-1302.

Gupta A, Dimeloe S, Richards DF, et al. Defective IL-10 expression and in vitro steroid-induced IL-17A in paediatric severe therapy-resistant asthma. Thorax 2014; 69: 508-515.

Ventura I, Vega A, Chaçon P, et al. Neutrophils from allergic asthmatic patients produce and release metalloproteinase-9 upon direct exposure to allergens. Allergy 2014; 69: 898-905.

Desai D, Gupta S, Siddiqui S, et al. Sputum mediator profiling and relationship to airway wall geometry imaging in severe asthma. Respir Res 2013; 14: 17

Chaudhuri R, McSharry C, Brady J, et al. Low sputum MMP-9/TIMP ratio is associated with airway narrowing in smokers with asthma. Eur Respir J 2014; 44: 895-904.

Elliot JG, Jones RL, Abramson MJ, et al. Distribution of airway smooth muscle remodelling in asthma: relation to airway inflammation. Respirology 2015; 20: 66-72.

Heijink IH, Nawijn MC, Hackett TL. Airway epithelial barrier function regulates the pathogenesis of allergic asthma. Clin Exp Allergy 2014; 44: 620-630.

Gielen V, Sykes A, Zhu J, et al. Increased nuclear suppressor of cytokine signaling 1 in asthmatic bronchial epithelium suppresses rhinovirus induction of innate interferons. J Allergy Clin Immunol 2015; 136: $177-188$.

$52-58$. disease. Eur Respir J 2015; 45: 1150-1162.

63 Blume C, Swindle EJ, Dennison P, et al. Barrier responses of human bronchial epithelial cells to grass pollen exposure. Eur Respir J 2013; 42: 87-97.

64 Lloyd CM, Saglani S. Asthma and allergy: the emerging epithelium. Nat Med 2010; 16: $273-274$.

65 Holgate ST. The airway epithelium is central to the pathogenesis of asthma. Allergol Int 2008; 57: 1-10

66 Spann KM, Baturcam E, Schagen J, et al. Viral and host factors determine innate immune responses in airway epithelial cells from children with wheeze and atopy. Thorax 2014; 69: 918-925.

67 Hirota JA, Knight DA. Human airway epithelial cell innate immunity: relevance to asthma. Curr Opin Immunol 2012; 24: 740-746.

68 Bonnelykke K, Sleiman P, Nielsen K, et al. A genome-wide association study identifies CDHR3 as a susceptibility locus for early childhood asthma with severe exacerbations. Nat Genet 2014; 46: 51-55.

69 Moffatt MF, Gut IG, Demenais F, et al. A large-scale, consortium-based genomewide association study of asthma. N Engl J Med 2010; 363: 1211-1221.

70 Woodruff PG, Dolganov GM, Ferrando RE, et al. Hyperplasia of smooth muscle in mild to moderate asthma without changes in cell size or gene expression. Am J Respir Crit Care Med 2004; 169: 1001-1006.

71 Shkumatov A, Thompson M, Choi KM, et al. Matrix stiffness-modulated proliferation and secretory function of the airway smooth muscle cells. Am J Physiol Lung Cell Mol Physiol 2015; 308: L1125-L1135.

72 Brightling CE, Bradding P, Symon FA, et al. Mast-cell infiltration of airway smooth muscle in asthma. $N$ Engl J Med 2002; 346: 1699-1705.

73 Kaur D, Gomez E, Doe C, et al. IL-33 drives airway hyper-responsiveness through IL-13-mediated mast cell: airway smooth muscle crosstalk. Allergy 2015; 70: 556-567.

74 Deshpande DA, Wang WC, McIlmoyle EL, et al. Bitter taste receptors on airway smooth muscle bronchodilate by localized calcium signaling and reverse obstruction. Nat Med 2010; 16: 1299-1304.

75 Orsmark-Pietras C, James A, Konradsen JR, et al. Transcriptome analysis reveals upregulation of bitter taste receptors in severe asthmatics. Eur Respir J 2013; 42: 65-78.

76 Grassin-Delyle S, Abrial C, Fayad-Kobeissi S, et al. The expression and relaxant effect of bitter taste receptors in human bronchi. Respir Res 2013; 14: 134.

77 Girodet PO, Dournes G, Thumerel M, et al. Calcium channel blocker reduces airway remodeling in severe asthma. A proof-of-concept study. Am J Respir Crit Care Med 2015; 191: 876-883.

78 Christianson CA, Goplen NP, Zafar I, et al. Persistence of asthma requires multiple feedback circuits involving type 2 innate lymphoid cells and IL-33. J Allergy Clin Immunol 2015; 136: 59-68.

79 Broekema M, Timens W, Vonk JM, et al. Persisting remodeling and less airway wall eosinophil activation in complete remission of asthma. Am J Respir Crit Care Med 2011; 183: 310-316.

80 Saglani S, Lui S, Ullmann N, et al. IL-33 promotes airway remodeling in pediatric patients with severe steroid-resistant asthma. J Allergy Clin Immunol 2013; 132: 676-685.

81 Kariyawasam HH, Pegorier S, Barkans J, et al. Activin and transforming growth factor-beta signaling pathways are activated after allergen challenge in mild asthma. J Allergy Clin Immunol 2009; 124: 454-462.

82 Mauri P, Riccio AM, Rossi R, et al. Proteomics of bronchial biopsies: galectin-3 as a predictive biomarker of airway remodelling modulation in omalizumab-treated severe asthma patients. Immunol Lett 2014; 162: 2-10.

83 Gao P, Simpson JL, Zhang J, et al. Galectin-3: its role in asthma and potential as an anti-inflammatory target. Respir Res 2013; 14: 136. 